\title{
BoSSA: The Deconstructed Violin Reconstructed
}

\author{
Dan Trueman ${ }^{1}$ and Perry R. Cook ${ }^{1,2}$ \\ ${ }^{1}$ Department of Music, Princeton University, Princeton, NJ 08544 \\ ${ }^{2}$ Department of Computer Science, Princeton University, Princeton, NJ 08544 \\ dan@music.princeton.edu_prc@cs.princeton.edu
}

\begin{abstract}
Traditional musical instruments provide compelling metaphors for human-computer interfacing, both in terms of input (physical, gestural performance activities) and output (sound diffusion). The violin, one of the most refined and expressive of traditional instruments, combines a peculiar physical interface with a rich acoustic diffuser. We have built a new instrument that includes elements of both the violin's physical performance interface and its spatial filtering audio diffuser, yet eliminates both the resonating body and the strings. The instrument, BoSSA (Bowed-Sensor-Speaker-Array), is an amalgamation and extension of our previous work with violin interfaces, physical models, and directional tonal radiation studies. In addition to describing the various physical and software elements that make up BoSSA, we discuss some of its musical features and potentials; we are particularly impressed by the sense of presence and intimacy it provides, and by its potential for creating a new kind of electronic chamber music.
\end{abstract}

\subsection{Introduction}

Traditional musical instruments provide compelling metaphors for human-computer interfacing, both in terms of input (physical, gestural performance activities) and output (sound diffusion). The violin, one of the most refined and expressive of traditional instruments, combines a peculiar physical interface with a rich acoustic diffuser.

Most previous efforts to integrate the violin with computing technology have focused on pitch detection, which, by itself, is not dependent on the physical interface (any audio signal will do). The bow has been used occasionally as a data input device [Paradiso and Gershenfeld 1997][Chafe 1999][Rose 1999], as has the fingerboard [Paradiso and Gershenfeld 1997]. All of these have been used in conjunction with traditional (or electric) stringed instruments and conventional speaker systems.

Various researchers have investigated the radiation properties of the violin [Bailey and Bissinger 1997] and the use of spherical speaker arrays [Caussé et al 1992][Wessel 1991][Roads 1996]. Our own recent research [Cook and
Trueman 1998, 1998b] has focused on creating a useable database of directional impulse responses from various stringed instruments. Combined with the creation of spherical multi-channel speaker arrays, this database allows us to model and manipulate the radiative timbral qualities of violins in traditional acoustic performance spaces. Until recently, these speaker arrays were used primarily with solid-body electric violins.

We have built a new instrument that includes elements of both the violin's physical performance interface and its spatial filtering audio diffuser, yet eliminates both the resonating body and the strings. The instrument, BoSSA (BowedSensor-Speaker-Array), is an amalga-mation and extension of our previous work with violin interfaces, physical models, and directional tonal radiation studies. It includes a sensor-bow (the RBow), a sensor-fingerboard (the Fangerbored), an array of bowed sensor-sponges (the Bonge), and a 12-channel spherical speaker array (the Critter). When combined with various synthesis and signal processing techniques, BoSSA offers the possibility for a new kind of electronic chamber music. 


\subsection{Input Interface Designs}

\subsection{The R-Bow}

The R-Bow (Figures 1 and 2) is a standard violin bow fitted with pressure and motion sensors. The pressure sensors (force-sensing-resistors (FSRs)) are mounted on light, soft foam at two locations between the stick and the hair. The resistance of FSRs is inversely proportional to the force applied, and is non-linear-their most sensitive region is at low pressures. This particular configuration of FSRs takes advantage of this sensitive low pressure region and makes for a highly responsive, playable instrument.

A dual axis accelerometer is mounted at the frog. This sensor provides relative position data on two axes: (1) as the player moves the bow from low string to high string and (2) as s/he tilts the bow forward and backward (or leans forward and backward). It can also be thought of as a motion sensor, indicating changes of velocity: (1) in the bowing direction and (2) perpendicular to the surface of the violin (up and down). These sensors are all wired to a belt-mounted 8-bit microcontroller which produces four MIDI continuous control messages corresponding to the four sensors (2 FSRs, 2 axes of the accelerometer). The technical requirements for using the R-Bow are minimal, and it has been used in many performances with few problems.
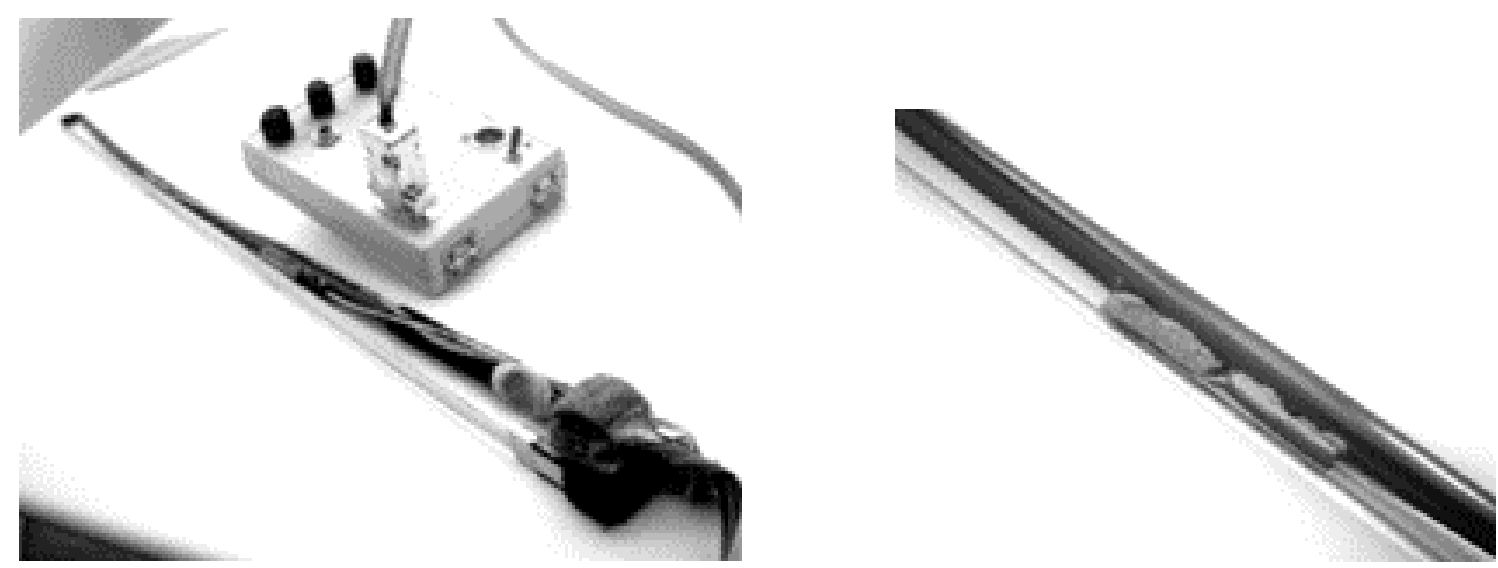

Figure 1. (left) R-Bow, and (right) one of the FSRs on the R-Bow.
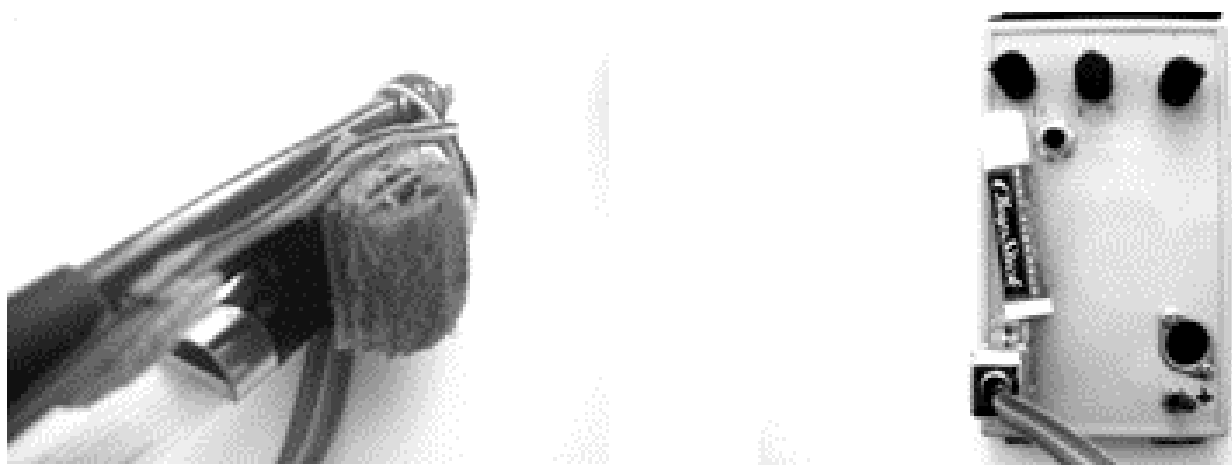

Figure 2. (left) R-Bow frog (with accelerometer) and (right) R-Bow belt-mounted computer (with Chapstick for scale). 


\subsection{The Fangerbored}

The Fangerbored is a violin fingerboard with a linear position sensor that can be played with traditional left-hand technique-a single string. It can be held by the right hand, and has four FSRs which can be played by the four fingers of the right hand (Figure 3). The right hand sensors-clearly not derived from the violin-are perhaps most like the holes on a Baroque flute, which have a range of "coveredness," from open to fully covered, that allow for subtle pitch manipulations. In addition, since the Fangerbored is free from a large resonating body, it has a dual axis accelerometer, creating two further expressive dimensions. The resulting instrument yields seven independent data streams: left-hand position (1), right hand finger pressure (4), and position/motion (2). Like the RBow, these sensors are wired to a microcontroller which converts the data into MIDI continuous control messages (except for the left-hand position sensor, which is converted to 14-bit pitchbend data).

\subsection{The Bonge}

The Bonge, motivated by the bowed string, consists of four bowed "sponges." Each "string" consists of a small piece of foam-covered wood resting freely between two fixed FSRs (Figure 4) and can be bowed, generating data that depends on direction, pressure, and bow speed; negative values indicate "up-bow," positive values indicate "down-bow." When bowed with the R-Bow, this instrument has eight dimensions: the four R-Bow dimensions and the four "strings" of the Bonge. A microcontroller converts the data to MIDI continuous control messages.

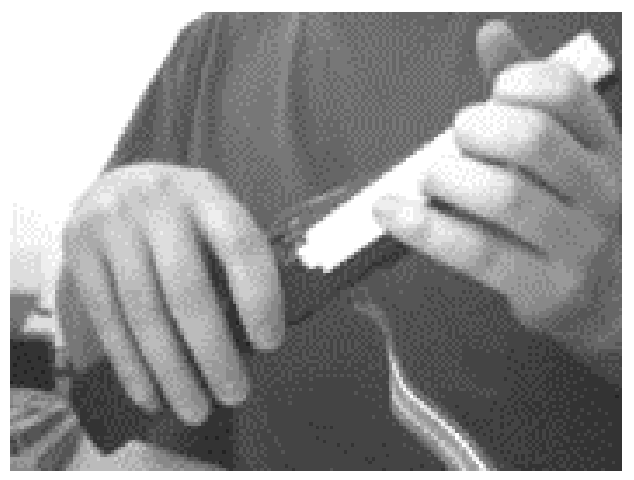

Figure 3. The Fangerbored, alone, and held in playing position.

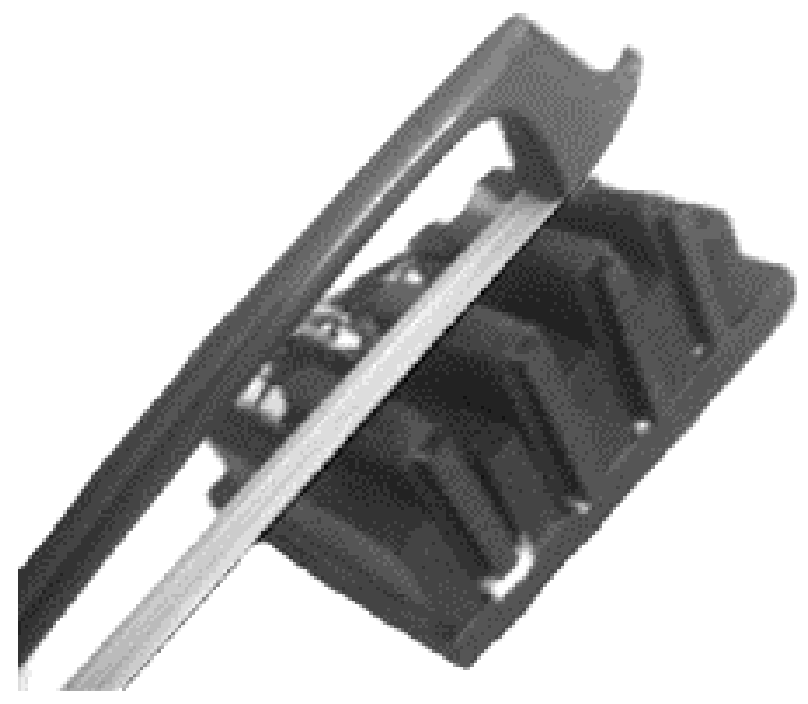

Figure 4. The Bonge; an array of bowed "sponges." 


\subsection{Output Interface Design}

The speaker array in BoSSA consists of 12 drivers arranged evenly over the surface of a sphere. In addition, each driver has its own insulated enclosure; the drivers are then isolated from one another and interact only minimally. Though the drivers are spaced symmetrically, the actual structure is not a sphere. It was necessary to construct a dodecahedron with flat faces so that the speakers could be mounted flush against the surface; it is important for the speakers to be sealed to improve their performance. The design consists of two 6-sided halves (made from poplar) each mounted on a central piece of pine (Figure 5).

The drivers currently in use with BoSSA are 3.5-inch coaxial car speakers. They claim to have a frequency response ranging from about $180 \mathrm{~Hz}$ to $16,000 \mathrm{~Hz}$. Though adequate, we hope to replace these eventually with higher quality drivers. Each driver enclosure is lined with acoustic fiber to minimize enclosure resonances. The speaker fits into a small tom-tom case and is easily transportable. It has an external patch-bay that allows it to be driven by $2,4,8$, or 12 channels, depending on the musical/instrumental needs. Software developed as part of our NBody project [Cook and Trueman 1998] allows us to simulate the directional tonal radiative qualities of violin, guitars, and other acoustic instruments with this speaker; it is, in a sense, an electronic substitute for the resonating body of the violin, a substitute that can be used with arbitrary resonances, including those of larger instruments like cellos and guitars.

\subsection{BoSSA}

\subsection{The Instrument}

Having deconstructed the violin into its primary constituent elements, we now reconstruct the instrument from its new electronic components (see Figure 6). BoSSA consists of the 12-channel spherical speaker array (the Critter) with the Bonge mounted on top and the Fangerbored mounted loosely on the side. When played with the R-Bow, BoSSA yields fourteen data streams: six from the Fangerbored (one of the accelerometer axes is rendered irrelevant by the mounting; the other is playable by rotating the Fangerbored up and down), four from the Bonge, and four from the R-Bow. This is more than a single player can play simultaneously; for practical purposes, the right hand "holes" on the Fangerbored are not usually played (assuming the right hand is holding the RBow), but can be used as switches by the left hand. Also, given that BoSSA is essentially stationary, it is impossible to bend over and take full advantage of the R-Bow accelerometers when bowing the Bonge. This effectively reduces the number of dimensions to nine, though there are other possible configurations that could reclaim a dimension or two.
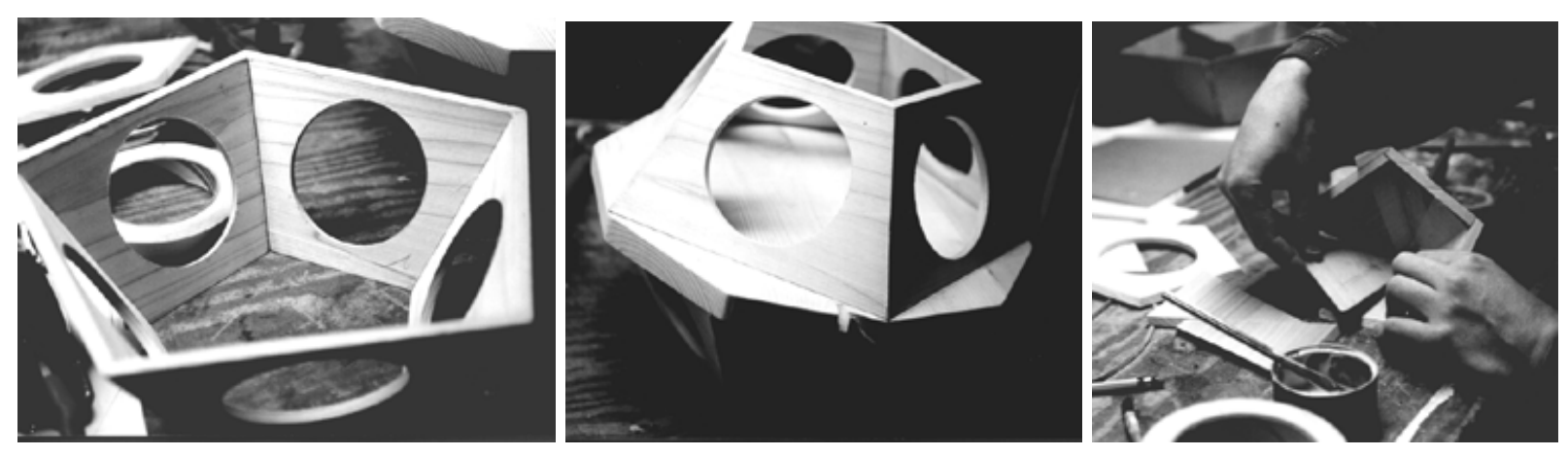

Figure 5. The BoSSA enclosure under construction; (left) one hemisphere, (center), before internal enclosures, (right) an internal enclosure. 

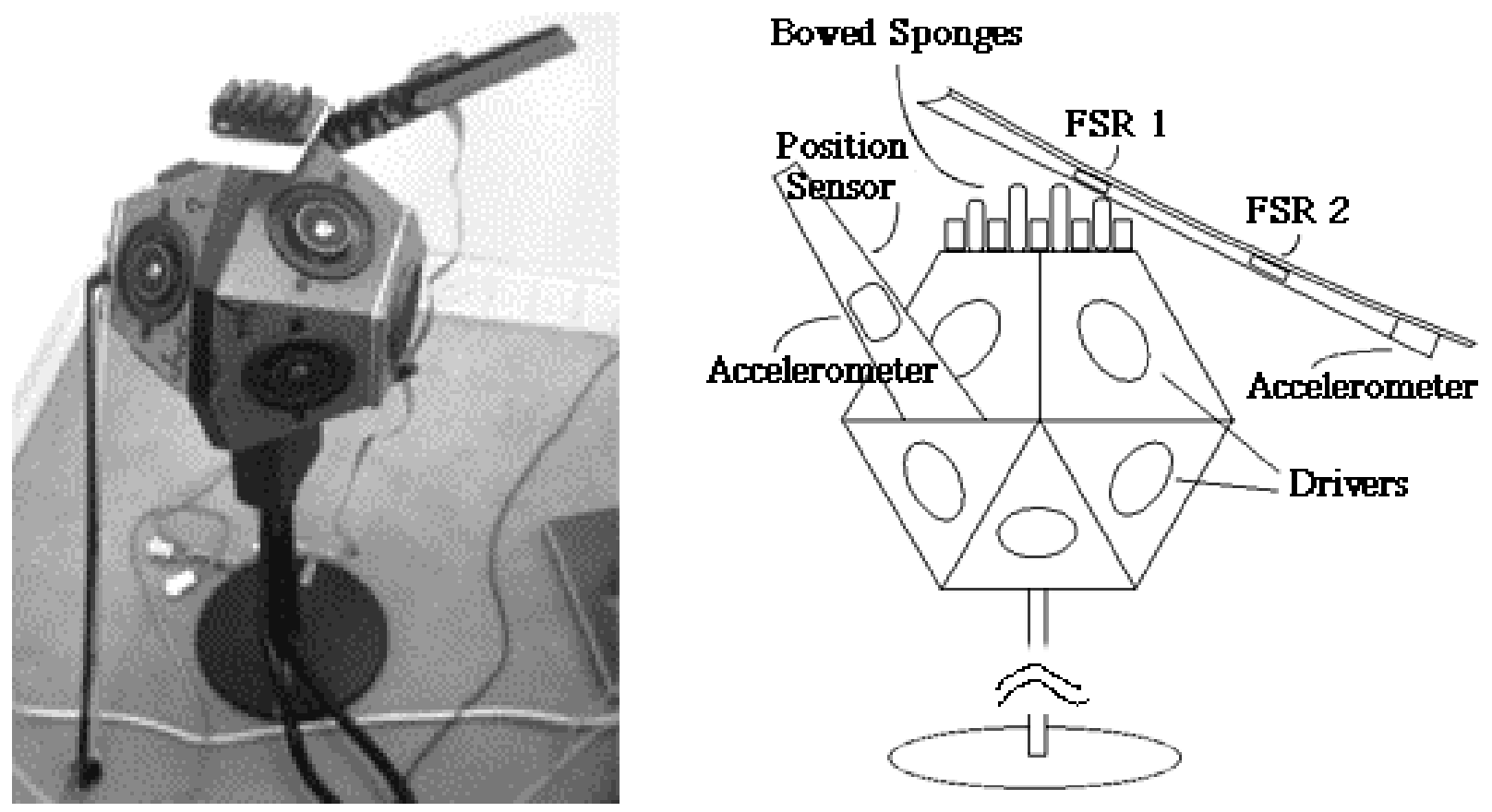

Figure 6. BoSSA (Bowed-Sensor-Speaker-Array).

\subsection{The Lobster Quadrille: A First Mapping}

The number of mappings from input sensors to output sounds is seemingly infinite. In its first performance, BoSSA was used with a relatively simple mapping that does not fully exploit its capabilities, but does provide a compelling example of its musical potential. The Lobster Quadrille is a setting of a poem from Lewis Carroll's Alice in Wonderland. Text was recorded (as read by Monica Mugan), separated into individual samples by sentence, and associated with the four "strings" of the Bonge. Down-bows played the sentences forward, up-bows played them backward. Left-hand finger position on the Fangerbored determined the relative pitch level of the samples; familiar violin left-hand techniques like glissandi, trills, and vibrato were possible.

The sentences were then sent through a 4voice bank of comb filters. The feedback coefficient - and hence the relative presence — of the comb filters was associated with the position of the Fangerbored; rotating the Fangerbored up resulted in rich comb filters, rotating it down left the samples essentially alone. A "chorale" was composed which controlled the pitches of the comb filters. By pressing one of the right hand "holes" on the Fangerbored, the performer could page through both the chords of the chorale and the sentences of the poem. During certain times in the piece, the Bonge would also supply a noise source to drive the comb filters, allowing reinforcement of the pitch content of the chorale. A model of bamboo wind chimes [Cook 1997] was attached to the accelerometers of the bow; when not bowing the Bonge, the performer could shake the chords. Finally, bow pressure was mapped to comb vibrato; when the performer leaned into the bow, the chords would gently oscillate. Each of the four comb filters and sentences were assigned to their own channel of drivers on the surface of the speaker, resulting in spatially diverse diffusion. All of this was executed in MAX/MSP on a PowerMac G3 300MHz, with a Mark of the Unicorn 2408 (MOTU) audio interface. The mapping and audio chain is illustrated in Figure 7. 
Audio Chain
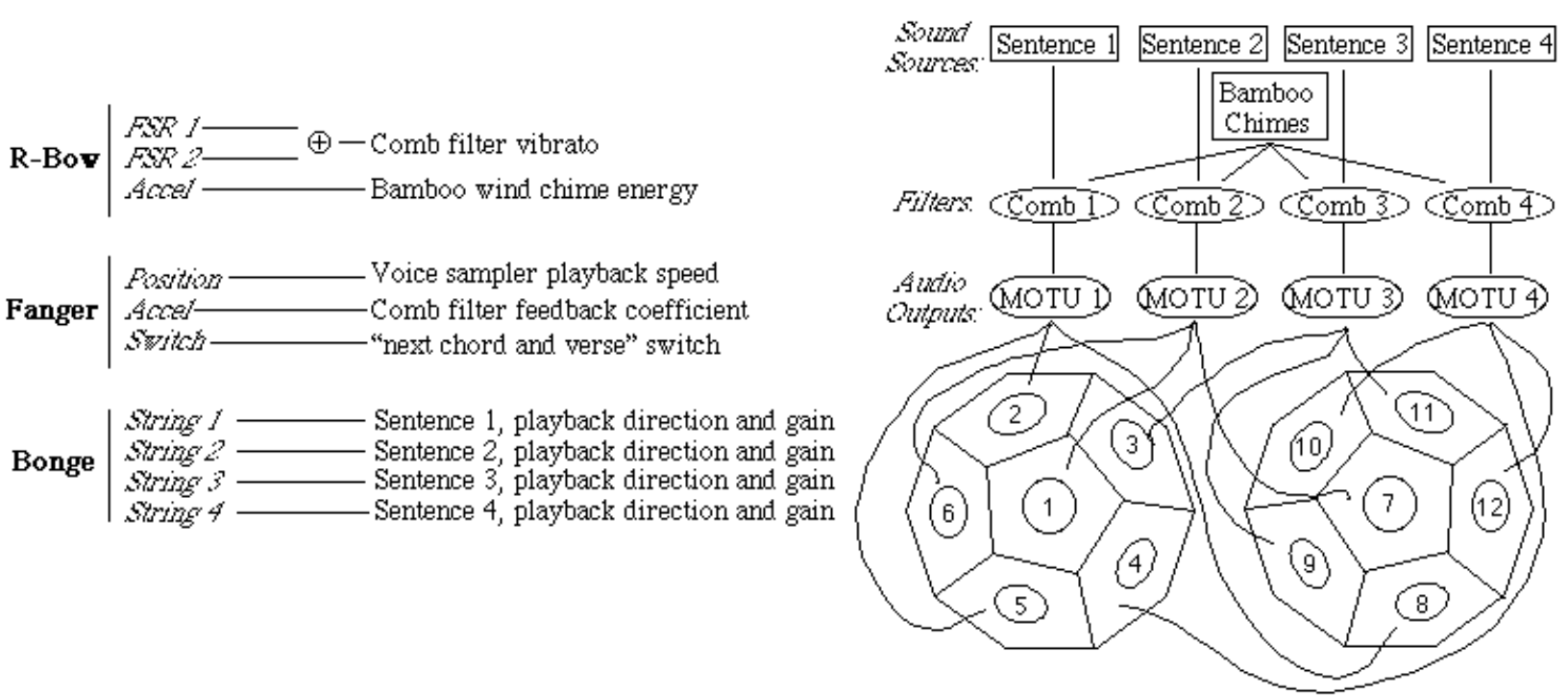

Figure 7. Illustration of sensor mappings (left) and audio signal path (right) for the Lobster Quadrille. In this 4-channel implementation, the outputs from the MOTU are interleaved and sent to drivers as far apart from one another as possible. The top face of the speaker is at left, the bottom at right. Drivers 1 and

7 are directly opposite one another, as are drivers $\mathrm{N}$ and $\mathrm{N}+6$.
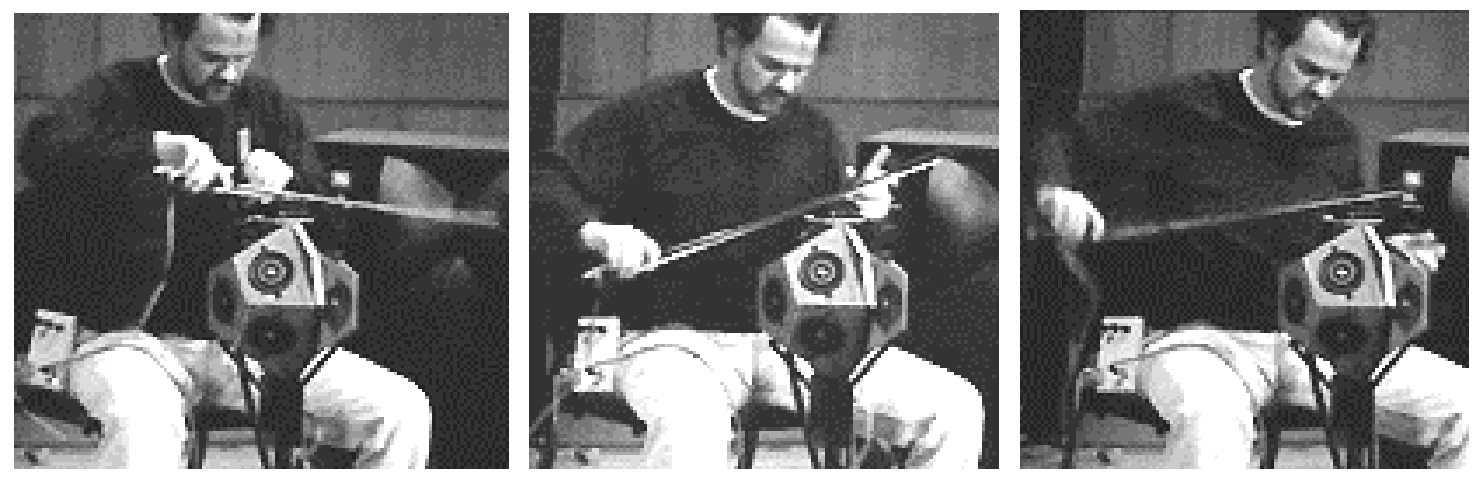

Figure 8. Three frames from a performance of the Lobster Quadrille. Note how the Fangerbored, which is used to "play" the comb filters, is rotated to different positions.

\subsection{Presence, Intimacy, Physicality, and Expression}

The unusual (in the computer music world) combination of input and output provided by BoSSA reorients the relationship between performer, sound source, and listener. Rather than relying on speakers disconnected from the performer, BoSSA places the sound source directly in the hands of the performer while still providing the enticing power and flexibility of software synthesis and signal processing techniques. And rather than using monodirectional speakers in single or multi-channel surround configurations, BoSSA takes advantage of the rich spatial diffusion qualities of outward-radiating spherical speaker arrays, again combined with the power and flexibility of digital signal processing.

There are two primary consequences of this reorientation. First, BoSSA provides a sense of instrumental presence that is taken for granted with traditional acoustic instruments, but is rare with electronic instruments. With BoSSA, there is a sense of the instrument being in the room with performer and listener in the same way as an 
acoustic instrument. The spherical radiation patterns engage the reverberant qualities of the room, reflecting off the walls and floors, surrounding performer and listener. Combined with the visual center of gravity created by the performer bowing the speaker, this sonic richness creates the focus of attention-a presence-that is so vital to instrumental performance practice. This also allows for a sort of intimacy that is difficult to achieve in computer music. We can now imagine inviting listeners in to a living room, asking them to "come closer, listen, look."

Second, the design of BoSSA demands an intensely physical relationship with sound. Not only is the sound source in our hands, requiring immediate attention and providing a short feedback loop, the instrument must be physically played, and with the notoriously challenging technique of the violin. It is our belief that the violin "as interface" has resulted in the creation of a rich tradition of physical performance technique that is not simply a compromise between human body and resonating body, but is in fact a natural and powerful expressive technique that can transcend the resonating body and be applied more generally in electroacoustic music. That it is difficult is one of its features, not flaws [Ryan 1992]. However, with BoSSA we are not primarily concerned with leaving this performance technique intact; rather we are building on and reinventing the traditional performance practice traditions of the violin in an electroacoustic context (for further discussion of the physical reinvention of the violin and violin technique that has occurred at various points in the history of the instrument, see [Trueman 1999]). As an example of this reinvention, the images of Figure 9 show a performer with the R-Bow (and an electric violin) assuming different positions in order to "play" the biaxial accelerometer. Harder to see, but equally significant, are the techniques created by playing the FSRs. With the R-Bow, it is possible to simply press down (and not play the string) to generate sound; this creates a realm of bow articulations that combines the traditional bowed string repertoire with new, electronically motivated techniques.
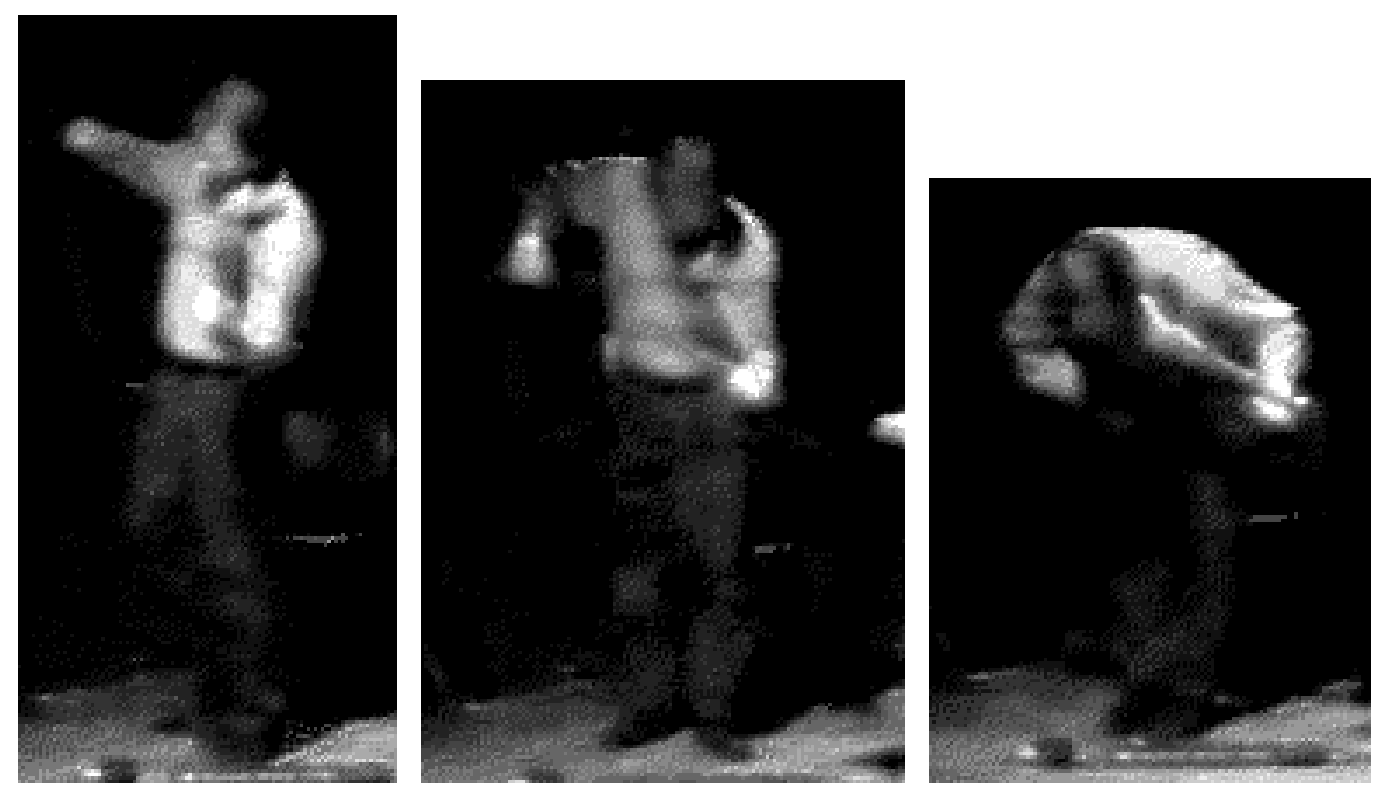

Figure 9. Three frames from a video of a performance with the R-Bow. To "play" the accelerometers, the performer moves the bow (and his whole body) into unusual positions.

This combination of presence, intimacy, and physicality presents an opportunity to create a new kind of electronic chamber music. Because it engages the reverberant qualities of the performance space, BoSSA blends better with acoustic instruments than conventional mono-directional speakers. Chamber music is, by definition, an intimate kind of music, and, because of its physicality, BoSSA lends itself to the kind of performance interaction that chamber musicians are accustomed to; we can move, cue, breathe, and draw attention similarly to the way a cellist might. 
This, in turn, offers new opportunities for expression and communication. According to the ethnomusicologist John Blacking, "music begins... as a stirring of the body" [Blacking 1973]. Blacking also extends Edward Cone's notion of "vicarious perfomance" [Cone 1968] by suggesting that "to feel with the body is probably as close as anyone can ever get to resonating with another person;" if our vicarious performance (as a listener, or fellow chamber musician) is so successful that we actually feel a "stirring" in our bodies, some sort of deep communication has taken place. BoSSA encourages this kind of physicality, both in terms of performance and listening.

\subsection{Future Generations}

It is easy to imagine an enormous family of instruments like BoSSA, instruments with input interfaces inspired by every known existing instrument, and with speaker arrays of various shapes and sizes. There are several developments that would aid in the creation of more BoSSA-like instruments: 1) lighter and higher quality speaker drivers (and perhaps different materials, like electro-static speakers); 2) lighter and smaller multi-channel amplifiers; 3) faster, easy to program microcontrollers; 4) lower latency software/hardware audio management (our current system features a manageable $\mathrm{I} / \mathrm{O}$ audio latency of about $10 \mathrm{~ms}$ ); 5) more software synthesis techniques with varied parametric controls; 6) force-feedback sensor mechanisms. The current bottleneck in terms of performance practicality is the weight, size and price of amplifiers; to run a full 12-channel system that is reasonably portable and inexpensive is currently impossible. Since power is not a primary need, it should be possible to create a relatively small and inexpensive 8-12 channel amplifier; the increasing popularity of "home theatre" systems may make this a reality.

\subsection{Acknowledgements}

Thanks to Judy and Larry Trueman for their help in designing and constructing the BoSSA speaker enclosure. Thanks to Monica Mugan for lending her voice to the Lobster Quadrille. This work supported in part by Intel, Interval Research, and the Arial Foundation.

\subsection{References}

Bailey, M. and Bissinger, G., 1997, "Measurement of direct radiation from a violin compared with spatial average of bridge force hammer-excited direct radiation," Proceedings of the Acoustical Society of America Conference, State College, Pennsylvania, Paper 4aMU4, Abstract only.

Blacking, J., 1973, "How Musical is Man," University of Washington Press, p. 111.

Caussé, R., Bresciani, J., and Warusfel, O., 1992, "Radiation of musical instruments and control of reproduction with loudspeakers," Proceedings of the International Symposium on Musical Acoustics, Tokyo.

Chafe, C., 1999, personal correspondence.

Cone, E., 1968, "Musical Form and Musical Performance," W.W. Norton.

Cook, P. and Trueman, D., 1998, "A Database of Measured Musical Instrument Body Radiation Impulse Responses, And Computer Applications for Exploring and Utilizing the Measured Filter Functions," Proceedings of the International Symposium on Musical Acoustics, Leavenworth, Washington.

Cook, P. and Trueman, D., 1998b, "NBody: Interactive Multidirectional Musical Instrument Body Radiation Simulations, and a Database of Measured Impulse Responses," Proceedings of the International Computer Music Conference, Ann Arbor.

Cook, P., 1997, "Physically Informed Sonic Modeling (PhISM): Synthesis of Percussive Sounds," Computer Music Journal, 21:3, pp. 3849.

Paradiso, J. and Gershenfeld, N., 1997, "Musical Applications of electric field sensing," Computer Music Journal, 21:2, pp. 69-89.

Roads, C., 1996, "The Computer Music Tutorial," MIT Press, Cambridge, p. 471.

Rose, J., 1999, URL: http://www.euronet.nl/users/ jrviolin/chaotic.html

Ryan, J., 1992, "Effort and Expression," Proceedings of the International Computer Music Conference, San Jose.

Trueman, D., 1999, "Reinventing the Violin," Ph.D. dissertation in music composition, Princeton University.

Wessel, D., 1991, "Instruments that Learn, Refined Controllers, and Source Model Loudspeakers," Computer Music Journal, 15:4, pp. 82-86. 